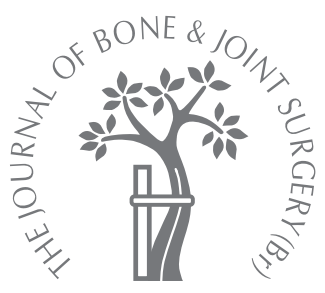

H. Dabasia, M. Kokkinakis, M. El-Guindi

From Stoke Mandeville Hospital, Aylesbury, England

\title{
Haematogenous infection of a resurfacing hip replacement after transurethral resection of the prostate
}

\begin{abstract}
A 66-year-old man presented with an infected resurfacing hip replacement in the immediate post-operative period following an uneventful transurethral resection of the prostate. Prophylactic antibiotics had been administered on induction of anaesthesia. The hip prosthesis had been inserted seven years previously and had been hitherto asymptomatic. The hip was washed out and microbiological examination identified Enterococcus faecalis as the infecting micro-organism.

Despite current recommendations, clinicians undertaking invasive procedures which can lead to bacteraemia in patients with prosthetic joint replacements should be aware of the risk of haematogenous seeding in such prostheses, which although low, has potentially disastrous consequences.
\end{abstract}

Infections of joint prostheses can be classified as early (presenting $<$ three months postoperatively), delayed (3 to 24 months) and late (> 24 months). ${ }^{1}$ In acute and delayed infections the pathogens responsible are usually introduced during the operation, whereas late infection occurs by haematogenous seeding of the implant from bacteraemia.

We present a case of late infection of a resurfacing hip replacement which occurred after transurethral resection of the prostate.

\section{Case report}

A 66-year-old man underwent uneventful transurethral resection of the prostate for urinary obstruction. Pre-operative mid-stream urine analysis revealed no bacteriuria and a prophylactic intravenous injection of $160 \mathrm{mg}$ of gentamicin had been administered on induction of anaesthesia according to local protocol. Histological examination of the excised prostatic tissue showed no evidence of malignancy.

He had undergone a resurfacing arthroplasty of the right hip seven years previously for osteoarthritis. The hip had been asymptomatic since then and no other significant comorbidities were identified.

Post-operatively he developed a fever and severe pain in the right groin and inability to bear weight on his right leg. He was systemically unwell and had marked limitation of movement of the right hip. The white blood cell count was $16.5 \times 10^{9} / 1$ and the CRP level was $312 \mathrm{mg} / \mathrm{l}$.
Urgent ultrasonography of the right hip showed a moderate collection of fluid within the joint and aspiration revealed frank pus. An open exploration was undertaken. No loosening of the prosthesis was noted. Intraoperative samples were sent for microbiological examination and a thorough debridement of the necrotic synovial tissue was followed by a voluminous washout with normal saline. The prosthesis was retained. The samples of fluid and tissue culture grew Enterococcus faecalis, sensitive to amoxicillin. He was started on intravenous amoxicillin, $2 \mathrm{~g}$ three times daily for six weeks, followed by oral amoxicillin $500 \mathrm{mg}$ three times daily for 12 months. When seen at three months he was systemically well. The right hip was painless with a full range of movement and he was fully weight-bearing. The white blood count and CRP levels were normal.

\section{Discussion}

There is limited information about late prosthetic joint infections which are widely regarded to be the result of haematogenous seeding. The incidence of haematogenous prosthetic joint infection has been described as $0.3 \%$ for large joint replacements, ${ }^{2}$ which equates to 210 of the 70000 hip replacements carried out annually in the United Kingdom. ${ }^{3}$ The presence of bacteraemia is a recognised risk factor in haematogenous seeding of prosthetic joints and, for Staphylococcus aureus bacteraemia, this risk has been shown to be as 
high as $34 \% .^{4}$ Common sources for such bacteraemia are the oral cavity, skin and respiratory, gastro-intestinal and urogenital tracts. A prospective study of 1000 patients followed up for six years after arthroplasty identified three cases of late infection, with recurrent skin infections being the likeliest source of bacteraemia. ${ }^{5}$

In our case the urinary tract was the source of bacteraemia, thereby leading to haematogenous seeding of the hip prosthesis. Urological procedures which involve instrumentation of the urinary tract are considered to be 'cleancontaminated'. ${ }^{6}$ In a multicentre, prospective study involving 284 patients after prostatectomy, 228 by transurethral resection and 54 by open surgery, $22 \%$ of the patients with a sterile urine culture pre-operatively developed bacteriuria post-operatively. This rose to $39 \%$ for those with preoperative bacteriuria. Bacteraemia secondary to bacteriuria occurred in one patient while another had an episode of primary bacteraemia. The presence of the American Society of Anaesthesiologists (ASA) grade $3 \geq{ }^{7}$ pre-operative urinary catheterisation with post-operative disconnection and/or replacement of the urinary catheter have been suggested as risk factors for infection after transurethral resection of the prostate. $^{8}$

Pre-operative systemic antibiotic prophylaxis aims to reduce the risk of local and systemic infection. The impact of bacteraemia is likely to be dependent on patient-related factors affecting the response to surgical infections, the source and level of this bacterial invasion and the potential morbidity of any infection.

The American Association of Orthopaedic Surgeons (AAOS) has published recommendations for antibiotic prophylaxis after urological procedures in patients with joint arthroplasties. ${ }^{9}$

Our patient received prophylactic antibiotics before transurethral resection of the prostate according to our hospital guidelines. We postulate that Enterococcus faecalis bacteraemia occurred from the urinary tract after completion of the procedure and the efficacy of the single dose of antibiotic had weakened, but before the innate physical barriers to infection in the prostatic bed had been restored. For similar cases, the extension of antibiotic prophylaxis throughout the period in which there is the potential for bacteraemia has been suggested. ${ }^{6}$

Although the cost, adverse side-effects and increased bacterial resistance associated with the widespread use of antibiotics are widely accepted, the impact of late prosthetic joint infection is devastating. Despite the current guidance suggesting that routine prophylaxis is not needed solely because of the presence of an implant, our case provides a valuable reminder that late haematogenous infection of prosthetic joints can still occur in an immunocompetent patient who has received pre-operative prophylaxis. An awareness of the risk, although rare, of late infection should be stressed on all patients who have prosthetic joint replacements. Clinicians need to be able to stratify the risk of bacteraemia in such patients when they are due to undergo invasive procedures and initiate prophylaxis of sufficient duration if this is deemed to be necessary.

No benefits in any form have been received or will be received from a commercial party related directly or indirectly to the subject of this article.

\section{References}

1. Zimmerli W. Infection and musculoskeletal conditions: prosthetic-joint-associated infections. Best Prac Res Clin Rheumato/ 2006;20:1045-63.

2. Little JW. The need for antibiotic coverage for dental treatment of patients with joint replacements. Oral Surg Oral Med Oral Pathol 1983;55:20-3.

3. Ghoz A, Macdonald D. (iii) New trends in total hip replacement: follow-up is it required and who pays? Curr Orthop 2008;22:173-6.

4. Murdoch DR, Roberts SA, Fowler VF, et al. Infection of orthopedic prostheses after Staphylococcus aureus bacteremia. Clin Infect Dis 2001;32:647-9.

5. Ainscow DA, Denham RA. The risk of haematogenous infection in total joint replacements. J Bone Joint Surg [Br] 1984;66-B:580-2.

6. Wolf JS Jr, Bennett CJ, Dmochowski RR, et al. Best practice policy statement on urologic surgery antimicrobial prophylaxis. J Urol 2008;179:1379-90.

7. Keats AS. The ASA classification of physical status: a re capitulation. Anesthesio/ogy 1978;49:233-6.

8. Girou E, Rioux C, Brun-Buisson C, Lobel B. The postoperative bacteriuria score: a new way to predict nosocomial infection after prostrate surgery. Infect Control Hosp Epidemiol 2006;27:847-54.

9. No authors listed. Association of Orthopaedic Surgeons. Information Statement: Antibiotic prophylaxis for urological patients with total joint replacements. http:// www.aaos.org (date last accessed 20 November 2008). 